\title{
Curva de Kuznets Ambiental para o Estado de Mato Grosso
}

\section{Environmental Kuznets Curve for the Mato Grosso State}

\author{
Rudolph Fabiano A. P. Teixeira* \\ Mario Augusto Bertella** \\ Luciana Togeiro de Almeida***
}

Resumo: As questões relativas ao desmatamento vêm ganhando destaque no debate ambiental mundial. Nesse cenário, o estado de Mato Grosso destaca-se de maneira negativa representando $36 \%$ do desmatamento acumulado na Amazônia brasileira. Com o objetivo de investigar a relação entre o desmatamento e o crescimento da renda, este trabalho estima uma Curva de Kuznets Ambiental (CKA) para 139 municípios de Mato Grosso. Usando dados para o ano de 2006, é estimada uma CKA para o desmatamento per capita contra a renda per capita, seu termo ao quadrado, a razão de bovinos pela área destinada à pecuária, a densidade demográfica, a extração de madeira per capita e os efeitos espaciais. Os resultados sugerem que existe uma CKA na forma de " $U$ " invertido. No entanto, ao se incorporar um termo cúbico para a renda, o crescimento econômico deixaria de expressar qualquer relação com o desmatamento dos municípios mato-grossenses.

Palavras-chave: Curva de Kuznets Ambiental. Desmatamento. Econometria espacial.

\begin{abstract}
Issues concerning deforestation have become a crucial theme in the environmental world debate. In this picture, Mato Grosso State has become an unfavorable example because it represents $36 \%$ of the accumulated deforestation in the Brazilian Amazon. In order to investigate the relationship between deforestation and income growth, this paper estimates an Environmental Kuznets Curve (EKC) for 139 cities of Mato Grosso through spatial econometrics. Using data for the year 2006, we estimate an EKC for the deforestation per capita against income per capita and other variables controlling the spatial effects. The preliminary results indicate that an EKC exists in a reversed U shape, i. e., the income growth reduces environmental effects from the maximum point. However, introducing a cubic

* $\quad$ Economista da Eletrobras e mestre em Economia pela Faculdade de Ciências e Letras de Araraquara (FCLAR) da Universidade Estadual Paulista (Unesp). E-mail: rudolphgp@hotmail.com

* * Professor doutor do Programa de Mestrado em Economia da Faculdade de Ciências e Letras de Araraquara (FCLAR) da Universidade Estadual Paulista (Unesp). E-mail: mabertella@yahoo.com.br

*** Professora doutora do Programa de Mestrado em Economia da Faculdade de Ciências e Letras de Araraquara (FCLAR) da Universidade Estadual Paulista (Unesp). E-mail: Itogeiro@gmail.com
\end{abstract}


term for the income, the economic growth would not reveal any relationship with the deforestation in the Mato Grosso State.

Keywords: Environmental Kuznets Curve. Deforestation. Spatial econometrics.

JEL Classification: Q00; C31.

\section{1 lntrodução}

O estado de Mato Grosso é o campeão do desmatamento acumulado na Amazônia Legal, com uma proporção de aproximadamente 36\% do total. Estimativas mostram que, entre 1988 e 2008 , foram desmatados 135 mil km² somente nesse estado, ou seja, uma área superior à soma dos territórios de Portugal (92 mil $\mathrm{km}^{2}$ ) e Holanda (41,5 mil km²), o que configura uma situação alarmante (PPCDMT, 2009). Em decorrência disso, estudos como o de Ferreira et al. (2008) trabalharam com a construção de cenários extremos, onde, mantidas as taxas de desmatamento do período 2003-2004, o estado de Mato Grosso contaria em 2020 com menos de $23 \%$ da cobertura florestal original, enquanto que, em 2033, essa mesma parcela seria reduzida para cerca de $10 \%$.

A literatura destaca o incremento da atividade pecuária (MARGULIS, 2003), a sojicultura (FEARNSIDE, 1999), e o corte de árvores (FEARNSIDE, 2003), como os principais vilões do processo de desmatamento no estado. Entre 1990 e 2008, o rebanho bovino em Mato Grosso sofreu um incremento de 9 para cerca de 26 milhões de cabeças, mantendo um ritmo de crescimento médio de aproximadamente 5,7\% ao ano. Paralelamente, a área plantada de soja no estado cresceu de 1,5 para 5,5 milhões de hectares no mesmo período, um incremento médio de 7,1\% ao ano. Já a extração de madeira em tora apresentou crescimento mais modesto, elevando-se de 16,8 mil metros cúbicos em 1990 para 20,2 mil metros cúbicos em 2008, um aumento médio de 0,97\% ao ano ${ }^{1}$ (SIDRA, 2009).

Em decorrência dos fortes impactos ambientais que Mato Grosso vem sofrendo, este trabalho se propõe a investigar a hipótese da Curva de Kuznets Ambiental (CKA) para o processo de desmatamento neste estado. Em outras palavras, buscase verificar empiricamente se a relação entre degradação ambiental (desmatamento) e crescimento econômico segue a forma de um " $U$ " invertido, indicando que, em municípios mais pobres, o incremento do produto estaria atrelado a uma piora do meio ambiente, enquanto que, em municípios mais ricos, o aumento do PIB seria responsável pela retração dos níveis de degradação ambiental.

1 É importante sublinhar que o Sistema IBGE de Recuperação Automática (SIDRA) fornece dados apenas sobre a extração legal de madeira. Dessa forma, o volume extraído de madeira em Mato Grosso, entre 1990 e 2008, provavelmente está muito aquém do verdadeiro nível. Segundo Fearnside (2003), em 1998, a Secretaria de Assuntos Estratégicos (SAE) calculou que 80\% do volume de toras cortadas nos estados da Amazônia eram ilegais. 
Nesse sentido, a principal contribuição do trabalho repousa na utilização da econometria espacial para estimar uma CKA em Mato Grosso, tendo em vista que, essa é a ferramenta mais indicada para tratar de dados georreferenciados. Como contribuições complementares podem ser citadas a elaboração de um modelo com dados mais atuais que o proposto por Prates (2008) e o enriquecimento da literatura sobre o tema, principalmente para estudos sobre a região Amazônica.

Além dessa breve introdução, o trabalho encontra-se dividido em mais cinco partes. A seção seguinte apresenta uma revisão da literatura da CKA; A seção 3 expõe a metodologia empregada. A seção 4 descreve a base de dados e o modelo empírico. A seção 5 corresponde à análise dos resultados. Por fim, a seção 6 traz as considerações finais.

\section{Revisão da Literatura}

Por volta dos anos de 1970, existia uma crença generalizada de que o crescimento econômico de uma nação seria o grande responsável pelos problemas ambientais, ou seja, era consenso que existia uma relação positiva entre crescimento econômico e a degradação do meio ambiente (FONSECA; RIBEIRO, 2005). No entanto, a partir da década de 1990, alguns economistas passaram a argumentar que tal visão era extremamente pessimista, na medida em que desconsiderava as inovações tecnológicas, a melhoria da educação, o progresso econômico e a evolução das instituições no desenvolvimento de uma nação, o que poderia minorar os problemas ambientais (STERN, 1998).

A discussão sobre a relação entre crescimento econômico e degradação ambiental permanece altamente controversa, debate que vem se enriquecendo com novas contribuições teóricas e evidências empíricas².

O trabalho de Grossman e Krueger (1991) foi um dos primeiros a trabalhar com evidências empíricas e buscar, por meio de modelos econométricos, estabelecer uma possível relação entre degradação ambiental e crescimento econômico. Os autores se utilizaram de uma amostra cross-country de três tipos de poluentes (dióxido de enxofre, fumaça e partículas suspensas), localizados em áreas urbanas de 42 países, juntamente com o PIB per capita (e seu termo ao quadrado) apurado nos mesmos. Considerando o dióxido de enxofre e a fumaça, encontrou-se uma relação na forma de "U" invertido com a renda per capita. Isto é, em baixos níveis de renda, a degradação do meio ambiente aumentaria com o crescimento do PIB per capita até certo limite (ponto de máximo) e, a partir daí, com o crescimento persistente do produto, os níveis de degradação passariam a declinar. Por sua vez, para o montante de partículas suspensas, a relação com a renda mostrou-se monotonicamente decrescente. A relação encontrada para os dois primeiros tipos

2 Vide, por exemplo, as contribuições de Brock e Taylor (2004), Igliori (2008) e Victor (2008).

TEIXEIRA, R. F. A. P.; BERTELLA, M. A.; ALMEIDA, L. T. Curva de Kuznets ambiental... 
de poluentes foi batizada de Curva de Kuznets Ambiental (CKA), tendo em vista a similaridade entre esta curva e aquela encontrada para o nível de renda e sua distribuição por Kuznets (1955).

Segundo Grossman e Krueger (1991, 1995), o respaldo teórico de tal suposição viria de três principais efeitos: escala, composição e tecnológico. O primeiro seria responsável pela parte ascendente da curva onde, em países pouco industrializados, a busca por melhorias nos níveis de emprego e renda seria prioritária se comparada a investimentos ambientais, tidos como bens de luxo. Por outro lado, o efeito composição atuaria no sentido de orientar o crescimento econômico para os setores nos quais os países são fatores-intensivo, ou seja, aqueles em que possuem vantagens comparativas com relação às demais nações, com a produção se dando de modo mais eficiente, poluindo menos. Já o efeito tecnológico responde pela modernização atrelada a um maior nível de produto. Esse efeito seria responsável pelo aumento da produção de forma mais eficiente no sentido ambiental.

Regidos por essa ideia, os países passariam por distintos estágios de desenvolvimento, orientados pela força de mercado e por mudanças institucionais. No primeiro estágio, marcado por uma transição de uma economia agrícola para uma industrializada, o crescimento econômico implicaria uma pressão cada vez maior sobre o meio ambiente, como resultado da ampliação do parque industrial. O estágio seguinte seria caracterizado pela maturação da sociedade e da infra-estrutura industrial, tendo em vista que, com as atividades básicas sendo atendidas, o crescimento de setores menos intensivos em recursos naturais e poluição passaria a ser prioritário. As melhorias tecnológicas começariam a mitigar o montante de matérias-primas e energia utilizado no processo produtivo, bem como a geração de resíduos e demais rejeitos. No último estágio de desenvolvimento, ocorreria o de-linking, isto é, o fim de qualquer relação entre o crescimento econômico e pressões ambientais (GROSSMAN; KRUEGER, 1995; SELDEN; SONG, 1994; SHAFIK; BANDYOPADHYAY, 1992).

Entretanto, alguns autores como De Bruyn et al. (1998) acreditam que a CKA não se sustenta no longo prazo. O formato de "U" invertido seria apenas o primeiro estágio de uma relação entre degradação ambiental e crescimento econômico. Isto porque, após certo nível de renda, existiria um novo ponto de inflexão (dado por um termo cúbico para a renda) que tornaria a trajetória da CKA novamente ascendente, com esta passando a ter uma forma similar a um "N", sugerindo que a degradação ambiental voltaria a aumentar em elevados níveis de renda per capita.

Assim, surgiram muitos outros trabalhos que se utilizaram de diferentes técnicas econométricas, e distintas amostras de dados, para investigar a existência de relações na forma de "U" invertido e "N" entre algum indicador de degradação ambiental e o PIB per capita, em uma dada localidade ou região. 
Shafik e Bandyopadhyay (1992) utilizaram dez indicadores de qualidade ambiental, a saber, ausência de água tratada, ausência de saneamento básico, partículas suspensas, níveis de dióxido de enxofre $\left(\mathrm{SO}_{2}\right)$, mudanças na área florestal, taxa anual de desmatamento, oxigênio dissolvido nos rios, coliformes fecais nos rios, lixo municipal per capita e emissões de gás carbônico per capita, para testar a hipótese da CKA em 149 países no período de 1960 a 1990.

Foram testados três modelos de dados em painel, a saber, log-linear, quadrático e cúbico, com o intuito de explorar não somente a relação entre a renda e cada um dos indicadores ambientais, mas também alguns outros fatores que poderiam agir como variáveis explicativas. Destarte, esses autores regrediram os indicadores ambientais contra a renda per capita, uma variável de tendência temporal para refletir as evoluções da tecnologia, e um termo de efeitos fixos para as características específicas de cada localidade.

Considerando todos os indicadores abordados, surgiram diferentes resultados. Para as variáveis: ausência de água tratada, ausência de saneamento básico, oxigênio dissolvido nos rios e coliformes fecais nos rios, os autores obtiveram uma relação negativa com o nível de renda. As medidas de desmatamento não revelaram relação com o produto per capita, ao passo que dois indicadores de poluição do ar (dióxido de enxofre e partículas suspensas) confirmaram a hipótese da CKA. Por sua vez, as emissões de carbono per capita e o lixo municipal per capita revelaram uma forte relação positiva com o crescimento do produto.

Nos primeiros anos após a publicação do estudo de Grossman e Krueger (1991), os trabalhos subsequentes basicamente utilizaram amostras de diferentes países, ou localidades em diferentes países, com dados cross-section ou em painel, para tentar verificar a hipótese da CKA, considerando os mais distintos indicadores de degradação ambiental (STERN, 1998). Entretanto, nenhum desses trabalhos considerou a hipótese de dependência espacial entre os distintos países, ou distintas localidades em diferentes países.

Stern (2000) foi um dos primeiros autores a estimar uma CKA considerando os aspectos espaciais da base de dados. Ele utilizou uma amostra de dados em painel com dependência espacial para 16 países da Europa Ocidental, durante o período 1960-1990. Como variável dependente, adotou-se a emissão de $S_{2}$; por sua vez, as variáveis explicativas utilizadas compreenderam a renda per capita, seu termo ao quadrado, além de uma variável de tendência de tempo. Os resultados indicaram a existência de uma CKA.

Maddison (2006), utilizando uma amostra de 135 países para os anos de 1990 e 1995, tentou verificar a hipótese de uma CKA global, buscando assumir os aspectos espaciais da base de dados. Utilizando como variáveis dependentes emissões de dióxido de enxofre $\left(\mathrm{SO}_{2}\right)$, monóxido de carbono (CO), óxidos de nitrogênio 
$\left(\mathrm{NO}_{X}\right)$ e uma mistura de componentes orgânicos, não foi encontrada uma forma de "U" invertido para nenhumas das equações apresentadas.

No Brasil, o recente trabalho de Carvalho e Almeida (2008) utilizou uma amostra cross-section de 187 países para o ano de 2004, assumindo um contexto de dependência espacial. Como variável dependente, foi utilizada a emissão de dióxido de carbono ou gás carbônico $\left(\mathrm{CO}_{2}\right)$ em toneladas métricas per capita. A renda per capita, seu termo ao quadrado, o consumo de energia per capita, as exportações per capita, e uma dummy para captar os países signatários do Protocolo de Kyoto foram tomadas como variáveis explicativas. O resultado encontrado foi uma CKA na forma de "U" invertido. Entretanto, ao se adicionar um termo cúbico para o PIB per capita, a CKA passou a exprimir uma forma de "N", ou seja, indicando que o crescimento persistente do nível de produto teria um novo ponto de inflexão acarretando posteriores aumentos dos níveis de degradação ambiental, conforme sugeriram De Bruyn et al. (1998).

Contudo, todos os trabalhos anteriores independentemente da técnica econométrica utilizada buscaram observar uma CKA considerando diversos países ou localidades através do mundo. Contrariamente a essa tendência, Vincent (1997) e Carson, Jeon e McCubbin (1997) argumentam que a CKA deveria ser estudada no contexto de um único país, tendo em vista que os dados de uma única nação são mais confiáveis de se avaliar do que a comparação de dados entre diferentes países $^{3}$. Além disso, as diferenças entre países pobres, em desenvolvimento e desenvolvidos sugerem que a renda não é normalmente distribuída entre as nações, mostrando que a mediana da renda per capita é um indicador melhor que a média do produto per capita em um contexto global ${ }^{4}$ (STERN, 1998).

Fonseca e Ribeiro (2005) e Lucena (2005) foram os pioneiros em verificar a hipótese da CKA para o Brasil. Os dois primeiros, utilizando-se de dados em painel referentes ao percentual de áreas estaduais preservadas nas 27 unidades da federação, durante os períodos de 1985, 1990, 1995 e 2000, encontraram resultados distintos, dependendo da forma funcional utilizada e do tamanho da amostra. O segundo estudo, por sua vez, considerando o Brasil como um todo, fez uso de uma amostra de série de tempo entre 1970 e 2003 utilizando emissões de $\mathrm{CO}_{2}$ como variável dependente. O autor não encontrou evidências de uma CKA.

3 Segundo Carson, Jeon e McCubbin (1997), a agregação de dados referentes a uma mesma variável oriunda de distintos países pode apresentar viés de diferentes naturezas como, por exemplo: distorções provocadas intencionalmente por algum interesse governamental, credibilidade da fonte geradora, forma como os dados são coletados, etc.

$4 \quad$ Na visão de Stern (1998), a média do produto per capita global possui uma distribuição assimétrica negativa, com os dados concentrados nos menores valores (já que a maior parte dos países possui reduzida renda per capita). De acordo com o autor, a mediana seria uma medida de tendência central mais apropriada, pois, refere-se, ao ponto em que a base de dados é dividida em duas partes iguais. 
De maneira mais específica, trabalhos recentes buscaram avaliar a hipótese da CKA para a Amazônia Legal brasileira. Araújo e Melo (2007) foram os primeiros a discutir a hipótese da CKA para a Amazônia brasileira. Os autores além de realizarem uma revisão da literatura, também fizeram sugestões de possíveis variáveis institucionais que poderiam ser relevantes para o caso da Amazônia como, por exemplo, a existência de secretarias municipais de meio-ambiente, ou a existência de legislação ambiental municipal. A presença dessas variáveis institucionais evidenciaria preocupações em âmbito municipal com a questão da degradação do meio-ambiente, principalmente, o desmatamento.

Gomes e Braga (2008), por sua vez, utilizaram dados sobre a taxa de desmatamento e a renda per capita nos estados da Amazônia Legal, durante o período 1990-2004, buscando verificar empiricamente a hipótese da CKA. Fazendo uso de uma modelagem de dados em painel para efeitos aleatórios, os autores estimaram dois modelos específicos de regressão. O primeiro se vale da forma quadrática tradicional, em que a taxa de desmatamento é a variável explicada e a renda per capita, seu termo ao quadrado, a densidade demográfica e o ano são as explicativas. O segundo modelo incorpora, além dessas variáveis, o termo cúbico da renda per capita. As estimações mostraram que para a função quadrática original da CKA, os resultados foram o inverso do esperado, isto é, foi obtida uma CKA na forma de "U". Para a função cúbica, a CKA apresentou o formato de "U" invertido, considerando um nível de renda inferior a $\mathrm{R} \$ 6.000$.

O trabalho de Santos et al. (2008) verificou a hipótese da CKA para 782 municípios da Amazônia Legal. Utilizando como variável dependente a área desmatada em hectares, e como variável explicativa o PIB per capita e seu termo quadrático, foi testada empiricamente, através de dados em painel, a relação entre crescimento econômico e impacto ambiental durante os anos de 2000 a 2004. As evidências apontaram para a existência da CKA, porém com modelos pouco representativos.

Ressalte-se que os três últimos trabalhos anteriormente citados buscaram avaliar a hipótese da CKA para o processo de desmatamento na Amazônia Legal, que vem gerando enormes preocupações não somente no Brasil, mas também no mundo todo. Dos nove estados que compõem a referida região - a saber: Acre, Amapá, Amazonas, Maranhão, Mato Grosso, Pará, Rondônia, Roraima e Tocantins, o estado de Mato Grosso é o que apresenta o maior índice de desmatamento acumulado, correspondendo a mais de um terço do desmatamento na Amazônia Legal. Nesse sentido, estudar o comportamento do desmatamento em Mato Grosso é primordial para se entender esse processo na Amazônia Legal e os seus consequentes impactos ambientais e sociais.

No que tange a estudos da CKA para o estado de Mato Grosso, Prates (2008) é o único trabalho conhecido até o momento. $O$ referido autor estimou um modelo de efeitos fixos com dados em painel para tentar explicar o desmatamento em 
Mato Grosso durante o período 2000-2004. Os resultados indicaram que renda e seu termo quadrático apresentaram os sinais esperados, contudo, essas variáveis não foram estatisticamente significantes. Por sua vez, das demais variáveis explanatórias utilizadas, o preço da soja, os gastos em agricultura, a dimensão do rebanho e o tamanho da população mostraram poder explicativo sobre o processo de desmatamento no estado.

A principal contribuição do presente trabalho reside na estimação de uma CKA para o estado de Mato Grosso, com o uso da econometria espacial. Segundo a literatura especializada esta é a ferramenta mais indicada para tratar de dados georreferenciados. Como contribuições secundárias, podemos citar a elaboração de um modelo com dados mais atuais que o proposto por Prates (2008) e o enriquecimento da literatura sobre o tema, principalmente, para estudos sobre a região Amazônica.

A forma funcional proposta possui o desmatamento per capita como variável a ser explicada em função da renda per capita, seu termo ao quadrado, seu termo cúbico, e demais variáveis explanatórias, controlando-se os efeitos espaciais. Isso se deve porque o processo de desmatamento ocorre independentemente do zoneamento legal de uma unidade geográfica. O corte de árvores (FEARNSIDE, 2003), o incremento da atividade pecuária (MARGULIS, 2003) e a densidade demográfica (RUPASINGHA et al., 2004) são alguns dos principais fatores destacados pela literatura que podem contribuir com o desmatamento em uma dada localidade.

Outro aspecto que procuramos destacar neste trabalho refere-se à estimação da CKA para um único estado brasileiro, no caso, Mato Grosso, expandindo o grau de desagregação proposto por Vincent (1997) e Carson, Jeon e McCubbin (1997). Esses autores afirmam que a CKA deve ser estimada para um único país em razão dos motivos já expostos, e esse artigo faz isso para um único estado da federação brasileira.

\section{Metodologia}

De modo geral, todo processo que se dá no espaço está sujeito à chamada Lei de Tobler, também conhecida como a Primeira Lei da Geografia, cujo enunciado pode ser estabelecido da seguinte forma: "tudo depende de todo o restante, porém o que está mais próximo depende mais do que aquilo que está mais distante". A Lei de Tobler destaca, com isso, o papel da proximidade para o estabelecimento da interação espacial entre os fenômenos. Como a dependência está muito ligada à interação espacial, é importante definir o que se entende por este conceito. De acordo com Odland (1988, p. 13), “[...] a interação espacial, que é o movimento de bens, pessoas ou informações através do espaço, significa que eventos ou circunstâncias num lugar podem afetar as condições em outros lugares se os lugares interagem entre si". 
A dependência espacial significa, por sua vez, que o valor de uma variável de interesse numa certa região $i$, digamos $y_{i}$, depende do valor dessa variável nas regiões vizinhas $j\left(y_{j}\right)$. É possível destacar três fontes primárias de dependência espacial: uma relacionada a uma variedade de processos de interação espacial, a saber, o processo de difusão espacial (difusão tecnológica, por exemplo), a troca de mercadorias entre as regiões (comércio), o comportamento estratégico (interdependência dos agentes nas regiões) e a dispersão ou espraiamento (a fronteira agrícola, por exemplo). Outra vinculada a erros nos dados espaciais, ocasionado pela falta ou baixa correspondência entre o escopo do fenômeno em estudo e o zoneamento das unidades espaciais (distritos, municípios, microrregiões, etc.) com respeito aos dados (ANSELIN, 1988). Finalmente, uma terceira, relacionada a erros de especificação do modelo. Pode-se denominar a primeira fonte como sendo autêntica e as duas outras como sendo espúrias.

O processo de desmatamento é muito sensível aos efeitos espaciais. A queima de florestas, o desenvolvimento de culturas agrícolas, a pecuária e o comércio clandestino de madeira são alguns fatores que se desenvolvem heterogeneamente no espaço, desencadeando processos de interação espacial, independentemente do zoneamento legal de uma unidade geográfica. Daí a importância da abordagem espacial para a análise econométrica da CKA para o estado de Mato Grosso.

\subsection{Análise Exploratória de Dados Espaciais (AEDE)}

Conforme destacam Perobelli et al. (2007), a análise exploratória de dados espaciais está baseada em aspectos espaciais da base de dados, tratando diretamente de dependência espacial (i.e. associação espacial) e heterogeneidade espacial. Em suma, a finalidade da AEDE é caracterizar a distribuição espacial, os padrões de associação espacial (clusters espaciais), verificar a ocorrência de diferentes regimes espaciais ou outras formas de instabilidade espacial (não estacionariedade) e identificar observações atípicas (i.e. outliers).

Para que a AEDE seja implementada de maneira eficiente, é necessária a utilização de variáveis intensivas ou espacialmente densas, já que variáveis absolutas podem induzir a enganos na análise. A utilização do total de observações em números absolutos de um fenômeno não é aconselhável, tendo em vista que estes atributos podem estar correlacionados com variáveis de escala, o que geraria correlações espaciais espúrias. Nesse sentido, a divisão da variável de interesse por algum indicador de intensidade como o número de habitantes ou o tamanho da área de uma região, por exemplo, soluciona esse problema (ANSELIN, 2005).

A AEDE é o método mais indicado para se obter medidas de autocorrelação espacial global e local, observando a influência dos efeitos espaciais por intermédio de instrumentos quantitativos e não pelo "olho humano" (ANSELIN, 1995). 


\subsubsection{Autocorrelação espacial global}

De acordo com Anselin (1988), um coeficiente de autocorrelação descreve um conjunto de dados que está ordenado em uma determinada frequência; assim, um coeficiente de autocorrelação espacial descreve um conjunto de dados que está ordenado em uma sequência espacial.

Uma forma de calcular a autocorrelação espacial global é por meio do I de Moran. Essa é uma estatística que fornece de maneira formal o grau de associação linear entre os vetores de valores observados em um tempo $t\left(z_{t}\right)$ e a média ponderada dos valores dos seus vizinhos, ou as defasagens espaciais $\left(W z_{t}\right)$, como são mais conhecidos na literatura. Valores do I de Moran maiores ou menores do que o valor esperado $E(I)=-1 /(n-1)$ revelam autocorrelação espacial positiva ou negativa, respectivamente (PEROBELLI et al. 2007).

Os valores dessa estatística variam entre -1 e +1 , onde -1 representa um coeficiente de correlação linear perfeitamente negativa e +1 representa um coeficiente de correlação linear perfeitamente positiva (ANSELIN, 1995). Conforme Perobelli et al. (2007), essa estatística pode ser representada através da seguinte notação matricial:

$$
I_{t}=\left(\frac{n}{S_{O}}\right)\left(\frac{z_{t}^{\prime} W z_{t}}{z_{t}^{\prime} z_{t}}\right) t=1, \ldots n
$$

Nessa equação, $z_{t}$ é o vetor de $n$ observações para o ano $t$ na forma de desvio em relação à média. W é a matriz de pesos espaciais que pode ser definida como uma matriz quadrada em que cada célula $w_{i j}$ indica relação existente entre as regiões $i$ e $j$ em um sistema de $n$ regiões. A célula $w_{i j}$ é nula no caso das regiões não serem vizinhas, caso contrário o valor passa a ser $1 . S_{O}$ é igual a $\Sigma \Sigma w_{i j}$, significando que todos os elementos da matriz de pesos espaciais $W$ devem ser somados.

A matriz de pesos espaciais quando é normalizada na linha, isto é, quando a soma dos elementos da linha é igual a 1, fornece a expressão (1) da seguinte forma:

$$
I_{t}=\left(\frac{z_{t}^{\prime} W z_{t}}{z_{t}^{\prime} z_{t}}\right) t=1, \ldots n
$$

A literatura registra diversos modos de construir a matriz de pesos espaciais, entretanto, o critério utilizado baseia-se na distância geográfica. A ideia básica é que duas regiões próximas geograficamente têm maior interação espacial. Nesse aspecto, a matriz de $k$-vizinhos mais próximos, $k=3$, que é utilizada neste trabalho; trata-se de uma matriz binária cuja convenção de vizinhança está formulada na distância geográfica ${ }^{5}$ (medida em quilômetros ou milhas, por exemplo). Formalmente, conforme Almeida, Perobelli e Ferreira et al. (2008), tem-se:

$5 \quad$ Foram testadas matrizes de k-vizinhos que variavam entre 1 e 20 vizinhos mais próximos. Escolheu-se a matriz $k=3$ vizinhos mais próximos porque esta apresentou o maior valor do I de Moran, conforme sugere a literatura. 


$$
\left\{\begin{array}{l}
w_{i j}^{*}(k)=0 \text { se } i=j \\
w_{i j}^{*}(k)=1 \text { se } \quad d_{i j} \leq D_{i}(k) \text { e } w_{i j}(k)=\frac{w_{i j}^{*}(k)}{\sum_{j} w_{i j}^{*}(k)} \text { para } k=1, \ldots, n \\
w_{i j}^{*}(k)=0 \quad \text { se } \quad d_{i j}>D_{i}(k)
\end{array}\right.
$$

na qual $d_{i j}$ é a distância, medida pelo grande círculo, entre os centros das regiões $i$ e $j$, a fim de que esta região $i$ tenha $k$-vizinhos; $D_{i}(k)$ denota um valor crítico que define o valor de corte para cada região, isto é, a distância máxima para considerar regiões vizinhas à região $i$, onde regiões acima deste ponto não serão consideradas vizinhas da região em questão; $w_{i j}$ representam os pesos espaciais, ou a influência da região j sobre a região $i$ e $k$ representa o número de vizinhos adotados para cada região geográfica, no caso, $k=3$.

A estatística $c$ de Geary é uma outra medida de autocorrelação espacial global. Da mesma forma que o I de Moran, testa a aleatoriedade espacial. Sua fórmula é dada abaixo:

$$
c=\frac{n-1}{2 \sum_{i} \sum_{j} w_{i j}} \frac{\sum_{i} \sum_{j} w_{i j}\left(y_{i}-y_{j}\right)^{2}}{\sum_{i}\left(y_{i}-\bar{y}\right)^{2}}
$$

em que $n$ é o número de regiões; $y_{i}$ é a variável de interesse; $\bar{y}$ é a média dessa variável e $w_{i j}$ é o elemento da matriz de pesos espaciais.

$\mathrm{O} c$ de Geary possui interpretação diferente do coeficiente I de Moran, tendo em vista que se utiliza de uma medida distinta de covariância (Anselin, 1988). O valor do $c$ de Geary situa-se entre 0 e 2, ao passo que sua média teórica é 1 . Valores menores que seu valor esperado, isto é, entre 0 e 1 indicam autocorrelação espacial positiva, enquanto valores maiores que 1 indicam autocorrelação espacial negativa. Valores de $c$ entre 0 e 1 , estatisticamente significativos, indicam concentração espacial dos dados, ao passo que valores entre 1 e 2 indicam dispersão da variável de interesse (MCPHERSON; NIESWIADOMY, 2005).

Anselin (1995) considera que tanto o I de Moran quanto o c de Geary, por serem medidas de associação espacial global, não são capazes de revelar padrões de associação espacial local. Dessa forma, autocorrelação espacial local deve ser mensurada de outra forma.

\subsubsection{Autocorrelação espacial local}

O objetivo da autocorrelação espacial local é captar padrões de associação local (clusters ou outliers espaciais), que são geralmente ocultados pelas estatísticas de autocorrelação espacial global. A autocorrelação local pode ser calculada pela 
estatística $I_{i}$ de Moran local, também conhecida como Indicadores Locais de Associação Espacial (LISA) ${ }^{6}$.

Conforme enfatiza Anselin (1995), os indicadores LISA devem satisfazer a dois critérios:

a) esses indicadores devem possuir, para cada observação, uma indicação de clusters espaciais significantes de valores similares ao redor de cada observação, e b) o somatório dos indicadores LISA, em todas as regiões, deve ser proporcional ao indicador de autocorrelação espacial global. Dessa maneira, o coeficiente $I_{i}$ de Moran local pode ser expresso como:

$$
I_{i}=\frac{\left(y_{i}-\bar{y}\right) \sum_{j} w_{i j}\left(y_{j}-\bar{y}\right)}{\sum_{i}\left(y_{i}-\bar{y}\right)^{2} / n}
$$

onde $n$ é o número de regiões; $y_{i}$ é a variável de interesse; $\bar{y}$ é a média dessa variável; $y_{j}$ é a variável de interesse nas regiões vizinhas a $i$ e $w_{i j}$ é o elemento da matriz de pesos espaciais.

A interpretação para esta estatística é dada por quatro tipos de padrões espaciais: o padrão Alto-Alto (AA) indica regiões com elevados valores para a variável de interesse, sendo vizinhas de regiões com elevados valores para a mesma. O padrão Baixo-Baixo (BB) revela localidades com reduzidos valores para a variável em análise, sendo circundadas por localidades com baixos valores para a mesma. O padrão Baixo-Alto (BA) mostra localidades com baixos valores para a variável investigada, que são vizinhas de regiões com altos valores para a mesma. Por fim, o padrão Alto-Baixo (AB) caracteriza regiões com altos valores para a variável em estudo, que são ladeadas por outras com baixos valores para a mesma.

\subsection{O Modelo de Econometria Espacial}

Os modelos de econometria espacial procuram lidar com o tratamento da dependência espacial e da heterogeneidade espacial. A natureza multidimensional, que provoca a autocorrelação espacial entre os atributos de uma determinada região, torna o método dos mínimos quadrados ordinários (MQO) inadequado para estimar modelos econométricos espaciais. Em decorrência, as estimativas por este método serão inconsistentes e/ou ineficientes (ANSELIN, 1988).

Autores como Florax, Folmer e Rey (2003) e Anselin (2005) sugerem o seguinte procedimento para a especificação de um modelo que leve em conta a presença de autocorrelação espacial:

a) Estimar o modelo clássico de regressão linear por MQO;

$6 \quad$ No acrônimo em inglês Local Indicators of Spatial Association. 
b) Testar a hipótese de ausência de autocorrelação espacial devido a uma defasagem ou a um erro através do multiplicador de Lagrange para a defasagem espacial $\left(M L_{\rho}\right)$ e o multiplicador de Lagrange para o erro espacial $\left(M L_{\lambda}\right)$;

c) Caso ambos os testes sejam não significativos, deve-se utilizar o modelo clássico; caso contrário, segue-se o próximo passo;

d) Se ambos os testes forem significativos, deve-se verificar as versões robustas de ambos, ou seja, o multiplicador de Lagrange robusto para a defasagem espacial $\left(M L R_{\rho}\right)$ e o multiplicador de Lagrange robusto para o erro espacial $\left(M L R_{\lambda}\right)$. Caso $M L R_{\rho}>M L R_{\lambda}$, identifica-se o modelo de defasagem espacial como o mais apropriado. Caso contrário, $M L R_{\rho}<M L R_{\lambda}$, adota-se o modelo de erro espacial.

Nesse sentido, serão apresentados os dois modelos espaciais indicados anteriormente: o de defasagem e o de erro. Cabe lembrar que a variável dependente do modelo é o desmatamento (y), e as variáveis explanatórias são a renda, seu termo quadrático e cúbico, a quantidade de rebanhos, a quantidade de madeira extraída e a população, todas representadas por $(X)$. As variáveis espaciais Wy e $W \mu$ serão explicadas pelos respectivos modelos a seguir.

\subsubsection{Modelo de defasagem espacial}

Neste modelo, utiliza-se um termo para capturar um provável efeito de vizinhança, por exemplo, o desmatamento de uma dada região provocando efeitos em seus vizinhos. O modelo pode ser expresso da seguinte forma:

$$
y=\rho W y+X \beta+\varepsilon
$$

em que $y$ é um vetor $n$ por 1 de observações sobre a variável dependente; Wy é o vetor $n$ por 1 da defasagem espacial para a variável dependente, $\rho$ é o coeficiente autoregressivo espacial (um escalar); $X$ é uma matriz $n$ por $k$ de observações sobre as variáveis explicativas exógenas (mais a constante) com um vetor associado $k$ por 1 de coeficientes de regressão $\beta$ e $\varepsilon$ é um vetor $n$ por 1 de termos de erro aleatório distribuído idêntica e independentemente (iid), com média zero e variância constante.

De acordo com Anselin (1988), ao não se considerar explicitamente a defasagem espacial nesse modelo, ocorreria um problema da mesma magnitude da omissão de variável relevante, isto é, os coeficientes estimados por MQO das variáveis explicativas seriam tendenciosos. Para se evitar tal problema, métodos de estimação alternativos como o uso de variáveis instrumentais (VI) ou o método da máxima verossimilhança (MV) são recomendados.

\subsubsection{Modelo de erro espacial}

O modelo de erro espacial é apropriado quando as variáveis não incluídas no modelo e presentes nos termos de erro são autocorrelacionadas espacialmente. Neste, procura-se capturar efeitos não modelados que se manifestam no termo 
de erro. Geralmente, as mensurações destes efeitos são difíceis, por exemplo, o comércio ilegal de madeira que se espraia por todas as regióes do sistema em questão. Este modelo pode ser expresso da seguinte maneira:

$$
\begin{gathered}
y=X \beta+\mu \\
\mu=\lambda W \mu+\varepsilon
\end{gathered}
$$

onde o coeficiente $\lambda$ é o parâmetro do erro-regressivo espacial que acompanha a defasagem do termo de erro $W \mu$. Desse modo, a dependência espacial pode ser decorrente de efeitos não modelados que não foram aleatoriamente distribuídos através do espaço. A utilização de MQO na presença de erros não esféricos geraria estimativas ineficientes, apesar de justas. Nesse aspecto, o modelo de erro-espacial deve ser estimado pelo método da máxima-verossimilhança (MV) ou pelo método generalizado dos momentos (MGM) de Kelejian e Prucha (1999).

\section{Base de Dados e Modelo Empírico}

Este trabalho fez uso dos softwares GeoDa (GEODATA ANALYSIS) e SPACESTAT 1.91. A amostra utilizada contém dados (cross-section) sobre 139 municípios mato-grossenses, referentes ao ano de 2006. A escolha desse ano justifica-se por apresentar os dados mais recentes, até então, para todas as variáveis utilizadas no estudo. O quadro 1 sintetiza a descrição de todas as variáveis utilizadas neste trabalho?.

\begin{tabular}{|c|c|c|c|c|}
\hline Variável & Descrição & $\begin{array}{l}\text { Sinal } \\
\text { Esperado }\end{array}$ & Referencial Empírico & Fonte \\
\hline$D E S_{i}$ & $\begin{array}{l}\text { Logaritmo da razão entre a } \\
\text { área desmatada de um muni- } \\
\text { cípio e a sua população. }\end{array}$ & & $\begin{array}{l}\text { Reis e Guzmán (1992); } \\
\text { Fonseca e Ribeiro (2005); } \\
\text { Gomes e Braga (2008). }\end{array}$ & $\begin{array}{l}\text { INPE e } \\
\text { IPEA }\end{array}$ \\
\hline$P_{i}$ & $\begin{array}{l}\text { Logaritmo da razão entre o } \\
\text { produto interno bruto de um } \\
\text { município e a sua população. }\end{array}$ & + & $\begin{array}{l}\text { Grossman e Krueger (1991); } \\
\text { Selden e Song (1994); Fon- } \\
\text { seca e Ribeiro (2005). }\end{array}$ & IPEA \\
\hline$P I B 2_{i}$ & $\begin{array}{l}\text { Logaritmo do } P I B_{i} \text { elevado ao } \\
\text { quadrado. }\end{array}$ & - & $\begin{array}{l}\text { Grossman e Krueger (1991); } \\
\text { Fonseca e Ribeiro (2005). }\end{array}$ & IPEA \\
\hline
\end{tabular}

Quadro 1 - Descrição das variáveis utilizadas

(continua)

7 Apesar de uma parte da literatura frisar a importância da expansão das plantações de soja no processo de desmatamento em Mato Grosso, a utilização de uma variável para captar esse processo não se revelou significativa. Na verdade, a utilização dessa variável tornou todos os modelos testados não significantes. Tais resultados, provavelmente, são frutos da alta correlação entre a variável atrelada ao rebanho bovino e a variável atrelada às plantações de soja na região. 
(continuação)

\begin{tabular}{|c|c|c|c|c|}
\hline $\mathrm{PIB}_{i}$ & $\begin{array}{l}\text { Logaritmo do } \text { PIB }_{i} \text { elevado } \\
\text { ao cubo. }\end{array}$ & $*$ & $\begin{array}{l}\text { Grossman e Krueger (1991); } \\
\text { De Bruyn et al. (1998); } \\
\text { Fonseca e Ribeiro (2005); } \\
\text { Gomes e Braga (2008). }\end{array}$ & IPEA \\
\hline$R E B_{i}$ & $\begin{array}{l}\text { Logaritmo da razão entre } \\
\text { efetivo de cabeças de gado } \\
\text { em um município e as suas } \\
\text { áreas de pastagens. }\end{array}$ & + & $\begin{array}{l}\text { Reis e Guzmán (1992); } \\
\text { Prates (2008). }\end{array}$ & SIDRA \\
\hline$M A D_{i}$ & $\begin{array}{l}\text { Logaritmo da razão entre } \\
\text { a quantidade de madeira } \\
\text { extraída em um município e a } \\
\text { sua população. }\end{array}$ & + & Reis e Guzmán (1992). & $\begin{array}{l}\text { SIDRA } \\
\text { e IPEA }\end{array}$ \\
\hline$D D M_{i}$ & $\begin{array}{l}\text { Logaritmo da razão entre a } \\
\text { população de um município } \\
\text { e a sua área. }\end{array}$ & - & $\begin{array}{l}\text { Selden e Song (1994); } \\
\text { Rupasingha et al. (2004); } \\
\text { Gomes e Braga (2008); } \\
\text { Prates (2008). }\end{array}$ & $\begin{array}{l}\text { IPEA e } \\
\text { INPE }\end{array}$ \\
\hline
\end{tabular}

Fonte: Elaboração dos autores.

Nota: * Caso a variável PIB3 $3_{i}$ não seja estatisticamente significativa, a CKA apresentará o formato de " $U$ " invertido; entretanto, caso a mesma revele-se estatisticamente significativa e com sinal positivo, não se pode negar a hipótese de uma CKA em forma de "N".

O modelo empírico proposto, a seguir, considera $D E S_{i}$ como a variável dependente e as demais, como as explanatórias:

$$
\begin{gathered}
D E S_{i}=\beta_{0}+\rho W D E S_{i}+\beta_{1} P I B_{i}+\beta_{2} P I B 2_{i}+\beta_{3} P I B 3_{i}+\beta_{4} R E B_{i}+\beta_{5} M A D_{i}+\beta_{6} D D M_{i}+u_{i} \\
u_{i}=\lambda W u_{i}+\varepsilon_{i}
\end{gathered}
$$

na qual a dependência espacial, se significativa, será captada ou por $W D E S_{i}$, que é a defasagem espacial do logaritmo da variável dependente ou pelo termo de erro $u_{i}$ composto pela sua defasagem espacial, mais um termo de erro $\varepsilon_{i}$ com média zero e variância constante. As letras gregas referem-se aos parâmetros a serem estimados.

Para que a hipótese da CKA na forma de "U" invertido tenha validade, o coeficiente $\beta_{1}$ precisa ser positivo e significativo, o coeficiente $\beta_{2}$ precisa ter o sinal negativo e ser estatisticamente significativo e o coeficiente $\beta_{3}$ não deve ser significativamente diferente de zero. Caso $\beta_{3}$ apresente sinal positivo e seja estatisticamente significativo, a CKA apresentará a forma de "N". É importante também enfatizar que, caso nenhum dos coeficientes da renda se mostre estatisticamente significativos, não existirá qualquer relação entre o crescimento econômico e a degradação ambiental (SELDEN; SONG, 1994; SHAFIK; BANDYOPADHYAY, 1992).

De acordo com Margulis (2003), o incremento do desmatamento nos municípios de Mato Grosso estaria atrelado à expansão da atividade pecuária. O aumento do rebanho bovino em uma determinada área exerceria uma forte pressão sobre a fronteira florestal. Assim, espera-se que o sinal de $\beta_{4}$ seja positivo e estatisticamente 
significativo. Já um aumento na quantidade de madeira extraída em uma determinada localidade também atuaria no sentido de elevar o desmatamento (FEARNSI$\mathrm{DE}, 2003)$. Quanto maior o número de toras de madeira retiradas de uma região, maior o desmatamento. Destarte, espera-se que $\beta_{5}$ apresente um sinal positivo e revele-se estatisticamente significativo. Por fim, segundo Rupasingha et al. (2004), o incremento da densidade demográfica estaria atrelado a menores níveis de degradação ambiental. Áreas com reduzida densidade demográfica seriam responsáveis por elevados índices de desmatamento, já que seriam regiões de expansão da fronteira agrícola e de difícil fiscalização. Por sua vez, regiões de elevada densidade demográfica atuariam no sentido de preservar, e até mesmo ampliar as áreas de floresta, mormente porque regiões altamente industrializadas e urbanizadas estariam em busca de qualidade ambiental. Espera-se que $\beta_{6}$ apresente o sinal negativo.

\section{Análise dos Resultados}

Antes da aplicação de qualquer modelo, é importante verificar se a taxa de desmatamento de um determinado município mato-grossense sofre impacto da taxa de desmatamento de seus vizinhos. As estatísticas I de Moran e $c$ de Geary fornecem essa resposta, indicando o grau de autocorrelação espacial global no processo de desmatamento entre distintas localidades. A Tabela 1 resume os resultados desses indicadores.

Tabela 1 - Indicadores de Autocorrelação Espacial Global: I de Moran e c de Geary

\begin{tabular}{llllll}
\hline Indicador & Coeficiente & Média & Desvio - Padrão & z-valor & p-valor \\
\hline I de Moran & 0,3060 & $-0,007$ & 0,0621 & 5,0451 & 0,0000 \\
c de Geary & 0,6326 & 1,000 & 0,0678 & $-5,4180$ & 0,0000 \\
\hline
\end{tabular}

Fonte: Elaboração dos autores com base no programa SpaceStat.

O coeficiente de 0,3060 para o I de Moran revela que existe autocorrelação espacial global positiva entre o desmatamento em um município e seus vizinhos, ou seja, o desmatamento não ocorre de forma aleatória. Por sua vez, um coeficiente de 0,6326 para o $c$ de Geary também sugere a existência de autocorrelação espacial global positiva no processo de desmatamento entre os municípios matogrossenses, corroborando o resultado obtido pelo I de Moran.

Uma maneira de refinar as análises do I de Moran e do $c$ de Geary é através dos Indicadores Locais de Associação Espacial (LISA). Esta estatística é capaz de revelar padrões de agrupamentos espaciais significativos (clusters). Dessa forma, foi elaborada a estatística LISA para o desmatamento entre os municípios de Mato Grosso, conforme a figura 1. 


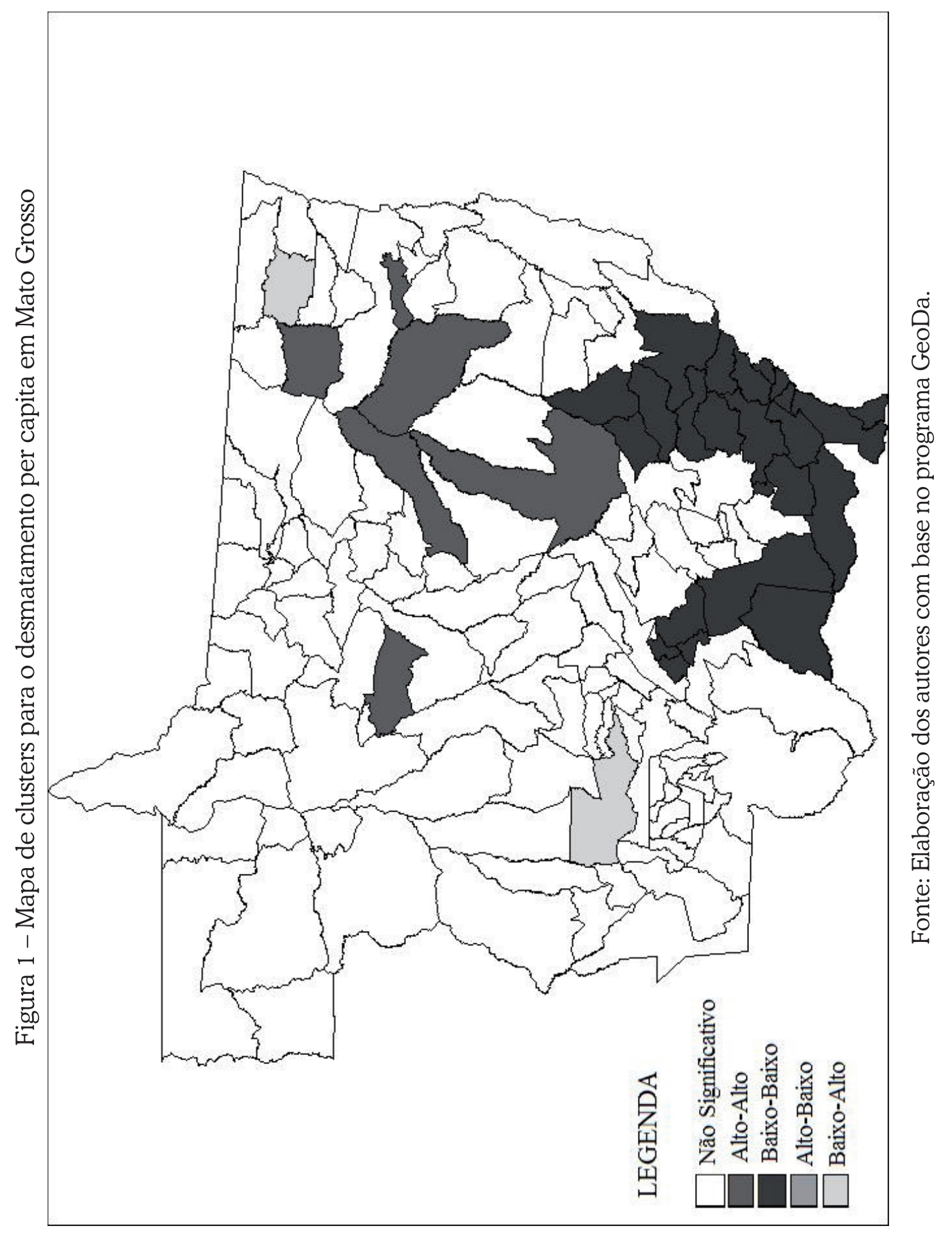


Os municípios em cinza chumbo estão presentes no padrão (Alto-Alto), já os municípios em preto referem-se ao padrão (Baixo-Baixo), por fim, as localidades em cinza claro representam o padrão (Baixo-Alto). Além disso, pode-se constatar que nenhum município apresentou o padrão (Alto-Baixo), na cor cinza escuro, no ano de 2006.

\subsection{Resultado dos Modelos Tradicionais}

O ferramental da AEDE detectou padrões espaciais significativos, ou seja, é possível constatar que o processo de desmatamento em Mato Grosso não é aleatório, existindo dois clusters espaciais significativos (AA) e (BB). Entretanto, para se investigar a hipótese da CKA para os municípios mato-grossenses, estimou-se, inicialmente, a equação (9) pelo método dos Mínimos Quadrados Ordinários (MQO) em busca do melhor modelo explicativo para uma possível CKA no estado de Mato Grosso. A Tabela 2 reporta os resultados obtidos.

Tabela 2 - Curva de Kuznets Ambiental (CKA): Resultados da Regressão por MQO

\begin{tabular}{lccc}
\hline Variáveis & Coeficientes & Modelo 1 & Modelo 2 \\
\hline Constante & $\beta 0$ & $-0,3887$ & $-0,4736$ \\
& & $(0,0043)$ & $(0,1647)$ \\
PIB & $\beta 1$ & 0,3245 & 0,4511 \\
& & $(0,0007)$ & $(0,3428)$ \\
PIB2 & $\beta 2$ & $-0,0737$ & $-0,1309$ \\
& & $(0,0004)$ & $(0,5354)$ \\
PIB3 & $\beta 3$ & & 0,0080 \\
& & & $(0,7856)$ \\
REB & $\beta 4$ & 0,0481 & 0,0475 \\
& & $(0,0103)$ & $(0,0121)$ \\
DDM & $\beta 5$ & $-0,0547$ & $-0,0545$ \\
& & $(0,0000)$ & $(0,0000)$ \\
MAD & $\beta 6$ & 0,0379 & 0,0379 \\
& & $(0,0055)$ & $(0,0056)$ \\
\hline Ponto de Máximo & & $\mathrm{R} \$ 9,038$ &
\end{tabular}

Fonte: Elaboração dos autores com base no programa SpaceStat.

Nota: Os valores entre parênteses referem-se às probabilidades.

O modelo 1 avalia a hipótese de uma CKA em forma de "U" invertido. Os coeficientes da renda per capita apresentaram os sinais esperados, sendo ambos significativos, não podendo se rejeitar essa hipótese. Por sua vez, o coeficiente referente à razão entre o rebanho de um município e suas áreas de pastagens (REB) revelou-se significativo, além de apresentar sinal positivo, o que já era esperado.

A razão da população de um município pela sua área, o coeficiente da variável (DDM), apresentou-se altamente significativo e com o sinal negativo. De 
acordo com Rupasingha et al. (2004), áreas com reduzida densidade demográfica seriam responsáveis por elevados índices de desmatamento, tendo em vista a baixa fiscalização e as possibilidades de expansão da fronteira agrícola. Já, regiões de elevada densidade demográfica atuariam no sentido de preservar e, até mesmo, ampliar as áreas de floresta considerando que sociedades industrializadas buscariam incrementar sua demanda por bens ambientais.

Por fim, o coeficiente relativo à razão entre a quantidade de madeira extraída em um município e a sua população (MAD) mostrou-se significativo e com sinal positivo, conforme o previsto. Esse resultado indica que quanto maior a extração de madeira per capita em uma dada localidade, maior é o grau de desmatamento que esta sofre. Além disso, o ponto de máximo encontrado para a renda per capita foi de $\mathrm{R} \$ 9.038,00$ a preços de 2000.

O segundo modelo estimado (modelo 2) analisa a hipótese de uma CKA em forma de "N". Para tanto, as variáveis renda per capita (PIB), seu termo ao quadrado (PIB2) e seu termo cúbico (PIB3) devem ser consideradas. Observando o modelo 2, é possível verificar que todos os coeficientes das variáveis atreladas à renda per capita apresentaram os sinais esperados, contudo, nenhum se mostrou estatisticamente significativo. Destarte, não se pode aceitar a hipótese de uma CKA com forma de "N" em Mato Grosso. Na verdade, ocorreria o fim de qualquer relação entre o crescimento econômico e pressões ambientais conforme sugerem Grossman e Krueger (1995), Shafik e Bandyopadhyay (1992), Selden e Song (1994), entre outros. Já as demais variáveis do modelo 2 se mostraram todas significativas e com o sinal esperado.

O passo seguinte consiste em averiguar as estatísticas de teste do multiplicador de Lagrange, segundo o procedimento de Florax, Folmer e Rey (2003) e Anselin (2005). De acordo a Tabela 3, tanto o multiplicador de Lagrange do ErroEspacial $\left(M L_{\lambda}\right)$ quanto o multiplicador de Lagrange da Defasagem-Espacial $\left(M L_{\rho}\right)$ mostraram-se estatisticamente significativos para os dois modelos analisados. Dessa forma, os multiplicadores de Lagrange do Erro e da Defasagem devem ser avaliados em suas versões robustas.

Tabela 3 - Diagnóstico da Regressão por MQO

\begin{tabular}{lcc}
\hline Estatisticas & Modelo 1 & Modelo 2 \\
\hline $\mathrm{R}^{2}$ & 0,4365 & 0,4369 \\
$\mathrm{R}^{2}$ _ajustado & 0,4154 & 0,4113 \\
LIK & 123,771 & 123,810 \\
AIC & $-235,542$ & $-233,620$ \\
SC & $-217,935$ & $-213,079$ \\
Teste Jarque - Bera & 33,7691 & 33,9779 \\
& $(0,0000)$ & $(0,0000)$ \\
\hline
\end{tabular}

(continua) 
(continuação)

\begin{tabular}{lcc}
\hline Estatisticas & Modelo 1 & Modelo 2 \\
\hline Teste Koenker - Bassett & 14,2207 & 15,6432 \\
& $(0,0143)$ & $(0,0158)$ \\
I de Moran & 0,3197 & 0,3198 \\
& $(0,0000)$ & $(0,0000)$ \\
ML - Erro & 25,7442 & 25,7498 \\
& $(0,0000)$ & $(0,0000)$ \\
ML - Defasagem & 10,6635 & 10,6127 \\
& $(0,0011)$ & $(0,0011)$ \\
MLR - Erro & 17,9672 & 18,1960 \\
& $(0,0000)$ & $(0,0000)$ \\
MLR - Defasagem & 2,8864 & 3,0588 \\
& $(0,0893)$ & $(0,0803)$ \\
\hline
\end{tabular}

Fonte: Elaboração dos autores com base no programa SpaceStat.

Nota: Os valores entre parênteses referem-se às probabilidades.

O multiplicador de Lagrange Robusto para o Erro-Espacial $\left(M R L_{\lambda}\right)$ mostrouse estatisticamente mais significativo do que o Multiplicador de Lagrange Robusto para a Defasagem-Espacial $\left(M R L_{\rho}\right)$. Assim, o modelo de erro espacial é tomado como o que melhor explica o processo de desmatamento entre os municípios de Mato Grosso.

Também é importante sublinhar que ambos os modelos (modelo1 e modelo 2) apresentaram o problema da não normalidade nos resíduos, como mostra o teste de Jarque-Bera. Assim, a estimação do modelo de erro-espacial para os dois modelos foi realizada através do método generalizado dos momentos (MGM) de Kelejian e Prucha (1999), que prescinde da hipótese de normalidade nos erros.

Outra constatação é que os modelos 1 e 2 apresentaram o problema da ausência de homocedasticidade, como indica o teste de Koenker-Bassett. Nesse sentido, as especificações dos modelos de erro-espacial foram modificadas para acomodar a heterocedasticidade na forma de grupos (groupwise heteroskedasticity).

\subsection{Resultados dos Modelos Espaciais}

A Tabela 4 expõe os resultados para os modelos de erro-espacial. Inicialmente, é possível verificar que o problema da ausência de homocedasticidade foi corrigido para os dois modelos propostos, isto é, 3 e 4, conforme o teste de KoenkerBassett. O modelo 3, que incorpora a dependência espacial na forma de erro para o modelo 1, apresentou os resultados esperados para todos os coeficientes. A variável renda per capita apresentou sinal positivo e seu termo ao quadrado o sinal negativo, com ambas sendo significativas. Por sua vez o coeficiente $\lambda$ mostrou-se altamente significativo, indicando que a dependência espacial no erro foi corrigida, e isto eleva a eficiência dos estimadores. 
Tabela 4 - Curva de Kuznets Ambiental (CKA): Resultados das Regressões por MGM

\begin{tabular}{lccc}
\hline Variáveis & Coeficientes & Modelo 3 & Modelo 4 \\
\hline Constante & $\beta 0$ & $-0,4281$ & $-0,5394$ \\
& & $(0,0004)$ & $(0,0723)$ \\
PIB & $\beta 1$ & 0,3553 & 0,5220 \\
& & $0,0000)$ & $(0,2134)$ \\
PIB2 & $\beta 2$ & $-0,0766$ & $-0,1521$ \\
& & $(0,0000)$ & $(0,4159)$ \\
PIB3 & $\beta 3$ & & 0,0106 \\
& & & $(0,6851)$ \\
REB & $\beta 4$ & 0,0471 & 0,0463 \\
& & $(0,0059)$ & $(0,0070)$ \\
DDM & $\beta 5$ & $-0,0601$ & $-0,0602$ \\
& & $(0,0000)$ & $(0,0000)$ \\
MAD & $\beta 6$ & 0,0378 & 0,0377 \\
& & $(0,0054)$ & $(0,0056)$ \\
WDES & $\lambda$ & 0,4644 & 0,4633 \\
& & $(0,0000)$ & $(0,0000)$ \\
Teste Koenker - Bassett & & 0,0528 & 0,0586 \\
& & $(0,8182)$ & $(0,8087)$ \\
\hline
\end{tabular}

Ponto de Máximo R $\quad$ R 10.167

Fonte: Elaboração dos autores com base no programa SpaceStat.

Nota: Os valores entre parênteses referem-se às probabilidades.

Com a presença explícita da dependência espacial no termo de erro, o ponto de inflexão da CKA eleva-se para $\mathrm{R} \$ 10.167,00$. Assim, deve-se enfatizar que, em modelos espaciais, o aumento da renda per capita atrelada ao ponto de máximo tem-se mostrado recorrente, como nos trabalhos de Rupasingha et al. (2004) e Carvalho e Almeida (2008).

O modelo 4, por sua vez, realiza a correção espacial na forma do termo de erro para o modelo 2. Novamente, todos os coeficientes dos termos ligados à renda apresentaram o sinal esperado para uma CKA com a forma de "N". Contudo, nenhum desses mostrou-se estatisticamente significativo. Em outras palavras, a renda não estaria atrelada ao processo de desmatamento em Mato Grosso. No mais, as demais variáveis deste modelo apresentaram o sinal esperado, além de se revelarem significativas, inclusive o coeficiente $\lambda$.

\section{Considerações Finais}

O presente trabalho procurou estimar uma CKA para o estado de Mato Grosso referente ao ano de 2006 controlando-se os efeitos espaciais. Nesse sentido, foram adotadas as duas formas funcionais mais utilizadas pela literatura em questão. 
A primeira, considerando a hipótese de uma CKA em forma de "U" invertido e a segunda, assumindo uma CKA em formato de "N".

Para a hipótese de uma CKA em forma de "U" invertido, foi utilizada como variável dependente o logaritmo do desmatamento per capita e, como variáveis independentes, o logaritmo da renda per capita, seu termo ao quadrado, o logaritmo da razão do rebanho bovino pela área de pastagem, o logaritmo da densidade demográfica e o logaritmo da extração de madeira per capita. Por sua vez, para a hipótese de uma CKA em formato de "N", todas essas variáveis anteriores foram mantidas, somando-se apenas o termo cúbico do logaritmo da renda entre as variáveis explicativas.

Entretanto, estudos que levam em consideração os aspectos espaciais da base de dados devem assumir explicitamente a autocorrelação espacial na especificação, na estimação e no teste de hipótese para dados em corte cruzado, como foi o caso. Assim, as estatísticas de teste do Multiplicador de Lagrange são capazes de revelar a melhor forma de incorporar a dependência espacial nas regressões, se, por meio da defasagem espacial da variável dependente ou por meio de um termo de erro que leve em conta as características espaciais.

Os testes de multiplicador de Lagrange indicaram que o melhor modelo, tanto para a hipótese de uma CKA em forma de "U" invertido quanto para uma CKA em forma de "N", foi o modelo de erro espacial. A modelagem pelo MGM de Kelejian e Prucha (1999), para uma CKA com dependência espacial na forma de "U" invertido (modelo 3), mostrou que todos os coeficientes foram significativos e revelaram os sinais esperados, inclusive o coeficiente $\lambda$. Foi, então, encontrado um ponto de máximo de $\mathrm{R} \$ 10.167,00$ a preços de 2000 , superior ao ponto encontrado para a estimativa por MQO de $\mathrm{R} \$ 9.038,00$, também a preços de 2000 .

Por sua vez, ao se estimar a hipótese de uma CKA em forma de "N" com efeitos espaciais, ou não, todos os coeficientes atrelados à renda tornam-se não significativos, indicando que a renda não possui relação com o processo de degradação ambiental. Por sua vez, as demais variáveis mostram-se significativas e com os sinais esperados.

Outro ponto a se destacar refere-se ao PIB per capita do estado de Mato Grosso. Este se encontrava em $\mathrm{R} \$ 7.330,00$ no ano de 2006, a preços de 2000 (IPEA, 2009). Nesse sentido, como o ponto de máximo encontrado para o modelo espacial em 2006 foi de $R \$ 10.167,00$ a preços de 2000, o estado de maneira geral ainda estaria na porção ascendente da CKA, ou seja, aumentos do desmatamento estariam atrelados a incrementos no nível de renda per capita.

Como sugestão de melhoria em trabalhos futuros, pode-se citar a importância de estimativas que incluam demais variáveis explicativas nas regressões para a hipótese da CKA em Mato Grosso, como: o impacto recente das plantações de cana-de-açúcar, a relevância da malha rodoviária estadual para o escoamento da 
produção e a presença de assentamentos rurais, o que pressionaria a demanda por terras com cobertura florestal. Outra linha interessante para trabalhos futuros é a estimação da CKA em Mato Grosso pelo método das regressões geograficamente ponderadas (RGP), uma das abordagens mais recentes em econometria espacial.

\section{Referências}

ALMEIDA, E. S; PEROBELLI, F. S; FERREIRA, P. G. C. Existe convergência espacial da produtividade agrícola no Brasil? Revista de Economia e Sociologia Rural, Brasília, v. 46, n. 1, p. 31-52, jan/mar, 2008.

ANSELIN, L. Spatial econometrics: methods and models. Boston: Kluwer Academic, 1988.

.Local indicators of spatial association - LISA. Geographical Analysis, Columbus, v. 27 n. 2, p. 93-115, Apr. 1995. Illinois, 2005.

Exploring spatial data with $G e o D a^{\mathrm{TM}}$ : a workbook. Urbana-Champaign: University of

ARAÚJO, P. T. M; MELO, A. S. Instituições ambientais da Amazônia Legal brasileira: um estudo das diferenças em nível municipal. In: ENCONTRO DA SOCIEDADE BRASILEIRA DE ECONOMIA ECOLÓGICA, 7., 2007, Fortaleza. Anais... Fortaleza: SBEE, 2007.

BROCK, W. A; TAYLOR, M. S. The green solow model. NBER Working Paper, Cambridge, MA, n. 10557, June 2004.

CARSON, R. T.; JEON, Y.; McCUBBIN, D. R. The Relationship between air pollution emissions and income: US data. Environment and Development Economics, Cambridge, v. 2, p. 433-450, 1997.

CARVALHO, T. S.; ALMEIDA, E. S. A hipótese da curva de Kuznets ambiental global: uma perspectiva econométrico-espacial. In: CONGRESSO DA SOCIEDADE BRASILEIRA DE ECONOMIA, ADMINISTRAÇÃO E SOCIOLOGIA RURAL, 46., 2008, Rio Branco. Anais... Rio Branco: SOBER, 2008.

DE BRUYN, S. M; VAN DEN BERGH, J. C. J. M; OPSCHOOR, J. B. Economic growth and emissions: reconsidering the empirical basis of environmental Kuznets curves. Ecological Economics, Amsterdam, v. 25, p. 161-175, May 1998.

FEARNSIDE, P. M. Combate ao desmatamento na Amazônia brasileira. Cadernos da Biodiversidade, Curitiba, v. 2, n. 2, p. 35-39, dez. 1999.

FEARNSIDE, P. M. Homem e ambiente na Amazônia. In: FEARNSIDE, P. M. (Org.). A Floresta Amazônica nas Mudanças Globais. Manaus, INPA, p. 1-18, 2003.

FERREIRA, D. A. C. et al. Mato, grosso até quando? Ciência Hoje, Rio de Janeiro, v. 42, n. 248, p. 26-31, maio 2008.

FLORAX, R. J. G. M.; FOLMER, H.; REY, S. J. Specification searches in spatial econometrics: the relevance of Hendry's methodology. Regional Science and Urban Economics, Amsterdam, v. 33, n. 5, p. 557-79, Sep. 2003.

FONSECA, L. N.; RIBEIRO, E. P. Preservação ambiental e crescimento econômico no Brasil. In: ENCONTRO DE ECONOMIA DA REGIÃO SUL, 7., 2005, Porto Alegre. Anais... Porto Alegre: ANPEC SUL, 2005. 
GOMES, S. C; BRAGA, M. J. Desenvolvimento econômico e desmatamento na Amazônia Legal: uma análise econométrica. In: CONGRESSO DA SOCIEDADE BRASILEIRA DE ECONOMIA, ADMINISTRAÇÃO E SOCIOLOGIA RURAL, 46., 2008, Rio Branco. Anais... Rio Branco: SOBER, 2008.

GROSSMAN, G.; KRUEGER, A. Environmental impacts of a North American free trade agreement. NBER Working Paper, Cambridge, MA, n. 3914, Nov. 1991.

GROSSMAN, G.; KRUEGER, A. Economic growth and the environment. Quarterly Journal of Economics, Massachusetts, v. 110, n. 2, p. 353-377, Feb. 1995.

IGLIORI, D. Deforestation, growth and agglomeration effects: evidence from agriculture in the Brazilian Amazon. Environmental Economy and Policy Research Working Papers, Cambridge, UK, n. 282008, 2008.

INSTITUTO BRASILEIRO DE GEOGRAFIA E ESTATÍSTICA. Sistema IBGE de Recuperação Automática: SIDRA. 2009. Disponível em: < http://www.sidra.ibge.gov.br/bda/> . Acesso em: 06 set. 2009.

INSTITUTO NACIONAL DE PESQUISAS ESPACIAIS. Projeto PRODES: Monitoramento da Floresta Amazônica Brasileira por Satélite. 2009. Disponível em: <http://www.obt.inpe.br/ prodes/index.html >. Acesso em: 25 abr. 2009.

INSTITUTO DE PESQUISA ECONÔMICA APLICADA. Ipeadata Regional. 2009. Disponível em:<http://www.ipeadata.gov.br/ipeaweb.dll/ipeadata?720284968>. Acesso em: 26 abr. 2009.

KELEJIAN, H. H.; PRUCHA, I. R. A generalized moments estimator for the autoregressive parameter in a spatial model. International Economic Review, Philadelphia, v. 40, n. 2, p. 509533, May 1999.

KUZNETS, S. Economic growth and income inequality. American Economic Review, Nashville, v. 45, n. 1, p. 1-28, Jan.1955.

LUCENA, A. F. P. Estimativa de uma Curva de Kuznets ambiental aplicada ao uso de energia e suas implicações para as emissões de carbono no Brasil. 2005. Dissertação (Mestrado em Ciências em Planejamento Energético) - Faculdade de Engenharia, Universidade Federal do Rio de Janeiro, Rio de Janeiro, 2005.

MADDISON, D. Environmental Kuznets Curves: A Spatial Econometrics Approach. Journal of Environmental and Management, London, v. 51, p. 218-230, 2006.

MARGULIS, S. Causas do desmatamento da Amazônia brasileira. Brasília: Banco Mundial, 2003.

MATO GROSSO. Secretaria de Estado do Meio Ambiente. Plano de ação para prevenção e controle do desmatamento e queimadas do Estado do Mato Grosso: PPCDMT. Cuiabá: SEMA, 2009.

MCPHERSON, M. A; NIESWIADOMY, M. L. Environmental Kuznets curve: threatened species and spatial effects. Ecological Economics, Amsterdam, v. 55, n. 3, p. 395-407, Nov. 2005.

ODLAND, J. Spatial autocorrelation. London: Sage, 1988.

PEROBELLI, F. S.; FERREIRA, P. G.; FARIA, W. R. Análise de convergência espacial do PIB per capita em Minas Gerais: 1975-2003. Revista Brasileira de Estudos Regionais e Urbanos, São Paulo, v. 1, n. 1, p. maio/out. 2007. 
PRATES, R. C. O desmatamento desigual na Amazônia Brasileira: sua evolução e consequências sobre o bem-estar. 2008. Tese (Doutorado em Ciências) - Escola Superior de Agricultura "Luiz de Queiróz", Universidade São Paulo, Piracicaba, 2008.

REIS, E. J; GUZMÁN, R. M. An econometric model of Amazon deforestation. Brasília: IPEA, 1992. (Texto para discussão IPEA, n. 265).

RUPASINGHA, A. et al. The environmental Kuznets Curve for US counties: a spatial econometric analysis with extensions. Regional Science, Urbana, Ill., v. 83, n. 4, p. 407-424, 2004.

SANTOS, R. B. N. et al. Estimativa da Curva de Kuznets Ambiental para a Amazônia Legal. In: CONGRESSO DA SOCIEDADE BRASILEIRA DE ECONOMIA, ADMINISTRAÇÃO E SOCIOLOGIA RURAL, 46., 2008, Rio Branco. Anais... Rio Branco: SOBER, 2008.

SELDEN, T. M.; SONG, D. Environmental quality and development: is there a Kuznets curve for air pollutions emissions? Journal of Environmental Economics and Management, New York, v. 27, n. 7, p. 1151-1160, Sep. 1994.

SHAFIK, N.; BANDYOPADHYAY, S. Economic growth and environmental quality: a time series and cross-country evidence. Policy Research Working Paper, Washington, n. 904, p. 1-55, June 1992.

STERN, D. I. Progress on the environmental Kuznets Curve? Environmental and Development Economics, Cambridge, v. 3, n. 2, p. 173-196, May 1998.

Applying recent developments in time series econometrics to the spatial domain. Professional Geographer, Washington, v. 52, n. 1, p. 37-49, Feb. 2000.

VICTOR, P. A. Managing without growth: slower by design not disaster. Cornwall: E. Elgar, 2008.

VINCENT, J. R. Testing for environmental Kuznets curves within a developing country. Environmental and Development Economics, Cambridge, v. 2, n. 4, p. 417-431, Mar. 1997.

Recebido em: 18/11/2010.

Aceito em: 23/10/2011. 
Editoração e impressão:

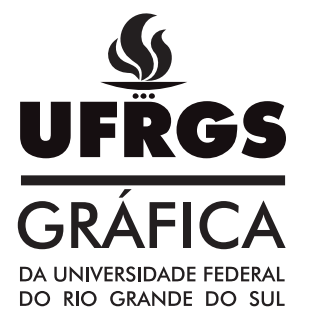

